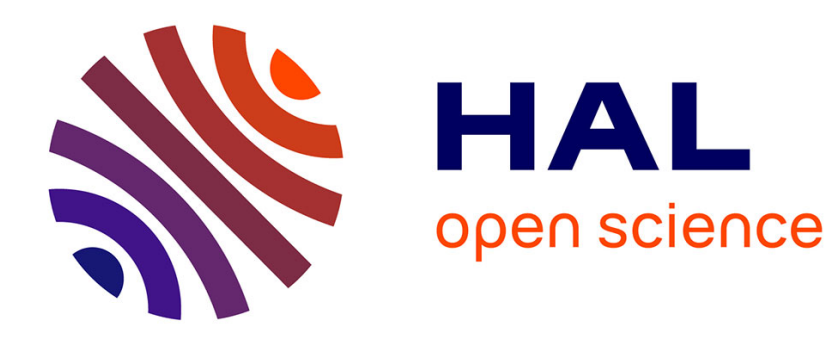

\title{
Un spectrophotomètre à double modulation
}

\author{
J.Y. Gay
}

\section{To cite this version:}

J.Y. Gay. Un spectrophotomètre à double modulation. Revue de Physique Appliquée, 1966, 1 (1), pp.32-36. 10.1051/rphysap:019660010103200 . jpa-00242681

\section{HAL Id: jpa-00242681 https://hal.science/jpa-00242681}

Submitted on 1 Jan 1966

HAL is a multi-disciplinary open access archive for the deposit and dissemination of scientific research documents, whether they are published or not. The documents may come from teaching and research institutions in France or abroad, or from public or private research centers.
L'archive ouverte pluridisciplinaire HAL, est destinée au dépôt et à la diffusion de documents scientifiques de niveau recherche, publiés ou non, émanant des établissements d'enseignement et de recherche français ou étrangers, des laboratoires publics ou privés. 


\title{
UN SPECTROPHOTOMÈTRE A DOUBLE MODULATION
}

\author{
Par J. Y. G\Y, \\ Laboratoire de l'École Supérieure de Physique, Faculté des Sciences, Marseille.
}

\begin{abstract}
Résumé. - On décrit une méthode de spectrophotométrie suivant laquelle les deux faisceaux sont modulés à des fréquences différentes, ce qui permet d'utiliser le même photorécepteur pour mesurer les deux flux.
\end{abstract}

Abstract. - A spectrophotometric method is described, in which the two both beams are modulated at different frequencies; the same photodetector is used for both the flux measurements.

I. Introduction. - Les lignes qui suivent décrivent la réalisation d'un spectrophotomètre qui, bien que destiné primitivement à l'étude de l'évolution rapide des couches minces, a cependant toutes les caractéristiques d'un spectrophotomètre classique.

Nous demandions à cet appareil d'avoir des caractéristiques qui répondent aux impératifs suivants :

$1^{0}$ Les deux flux (flux de mesure et flux de comparaison) devaient être mesurés simultanément au moyen du même photorécepteur afin d'éviter les erreurs éventuelles dues à la stabilité relative de deux récepteurs différents.

$2^{\circ}$ La mise au point devait être aussi simple que possible, ce qui excluait les systèmes à asservissement de gradation (déplacement d'un coin photométrique ou variation de largeur de fente).

Ces deux conditions nous ont conduit à adopter le principe de mesure décrit ci-après.

II. Principe de fonctionnement. - Les deux flux $\varphi_{1}$ et $\varphi_{2}$ (flux de mesure et flux de comparaison) sont modulés à deux fréquences différentes au moyen d'un dispositif convenable que nous décrirons plus loin. Grâce à un système optique, ils sont dirigés sur la même partie de la photocathode d'une cellule à multiplicateur d'électrons; par suite, ils donnent lieu dans le circuit anodique de cette cellule à deux courants dont les amplitudes sont respectivement proportionnelles à $\varphi_{1}$ et à $\varphi_{2}$, sous réserve que le photomultiplicateur travaille en régime linéaire.

La présence d'une résistance de charge dans le circuit d'anode permet d'obtenir deux tensions alternatives proportionnelles à ces courants. Il suffit alors de séparer ces deux tensions en jouant sur la différence qui existe entre leurs fréquences et de les envoyer sur un système d'enregistrement pour avoir une mesure simultanée de $\varphi_{1}$ et de $\varphi_{2}$. Cette séparation s'effectue au moyen d'un " discriminateur » filtrant dans le courant anodique chacune des deux composantes et restituant, sur deux sorties distinctes, des tensions redressées proportionnelles aux amplitudes de chacune des composantes intéressantes de la tension d'entrée.

III. Schéma synoptique de l'appareillage. - On peut décomposer l'ensemble du montage en deux parties principales, complétées par des dispositifs annexes nécessaires à son fonctionnement ( $f \mathrm{~g} g .1)$.

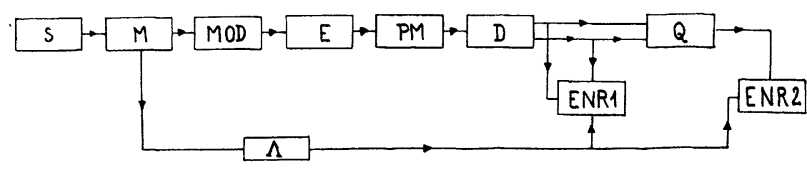

Fig. 1. - Schéma synoptique du spectrophotomètre. (S : source ; $\mathrm{M}$ : monochromateur ; MOD : modulateur double ; E : échantillon; PM : photomultiplicateur; D : discriminateur de signaux; ENR1: enregistrement $\mathrm{X}=\varphi_{1}(\lambda), \mathrm{Y}=\varphi_{2}(\lambda) ; \mathrm{Q}:$ dispositif de quotient ; ENR2 : enregistrement $X=\varphi_{1} / \vartheta_{2}(\lambda)$, $\mathrm{Y}=\lambda ; \Lambda$ : repérage des longueurs d'onde.)

a) Partie optique. - La partie optique comporte :

- l'ensemble source, monochromateur, substance en cours d'étude (dans le cas d'une spectroscopie d'absorption) ou couche mince déposée sur un support ;

- le dispositif modulateur destiné à différencier fréquentiellement les deux flux $\varphi_{1}$ et $\varphi_{2}$;

- diverses lentilles convergentes nécessaires à la conjugaison des différents éléments.

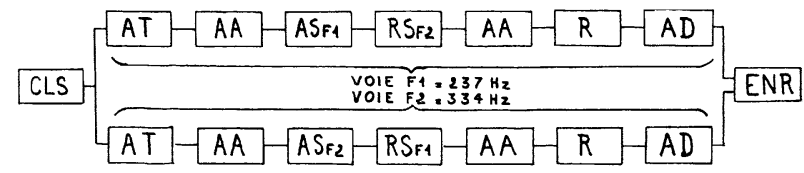

Fig. 2. - Schéma synoptique de la partie électronique réalisant la discrimination et la transformation des signaux. (CLS : conversion lumière-signal électrique ; AT : atténuation; AA : amplification apériodique ; ASF1 : amplification sélective à la fréquence F1; RSF2 : réjection sélective à la fréquence $\mathrm{F} 2$; $\mathrm{R}$ : redressement ; AD : adaptation ; ENR : enregistrement.) 
b) Partie électronique. - La partie électronique comporte :

- le transducteur flux lumineux-signal électrique (photomultiplicateur) ;

- l'ensemble électronique de discrimination et de transformation des signaux recueillis. Cet ensemble se compose de divers éléments indiqués sur la figure 2. ;

- l'enregistreur des mesures de $\varphi_{1}$ et $\varphi_{2}$ (table traçante XY par exemple).

Éventuellement, on peut prévoir l'adjonction de deux autres éléments :

- un dispositif quotientmètre ;

- l'enregistreur du quotient $\varphi_{1} / \varphi_{2}$.

Dans ce cas, l'étage d'enregistrement des deux flux $\varphi_{1}$ et $\varphi_{2}$ n'a plus de raison d'être.

Enfin, il est nécessaire de posséder un dispositif de repérage des longueurs d'onde de la lumière fournie par le monochromateur.

Les dispositifs annexes sont les suivants :

- alimentation de la source lumineuse ;

- alimentation en haute tension du photomultiplicateur ;

- alimentation en haute et basse tensions de la partie électronique.

IV. Description de l'appareillage. - 1. ModuLATION optique. - Au moyen d'un système optique convergent, nous conjuguons le plan de la fente de sortie du monochromateur avec le plan d'un disque transparent disposé perpendiculairement à la direction du flux lumineux et de telle sorte que l'image

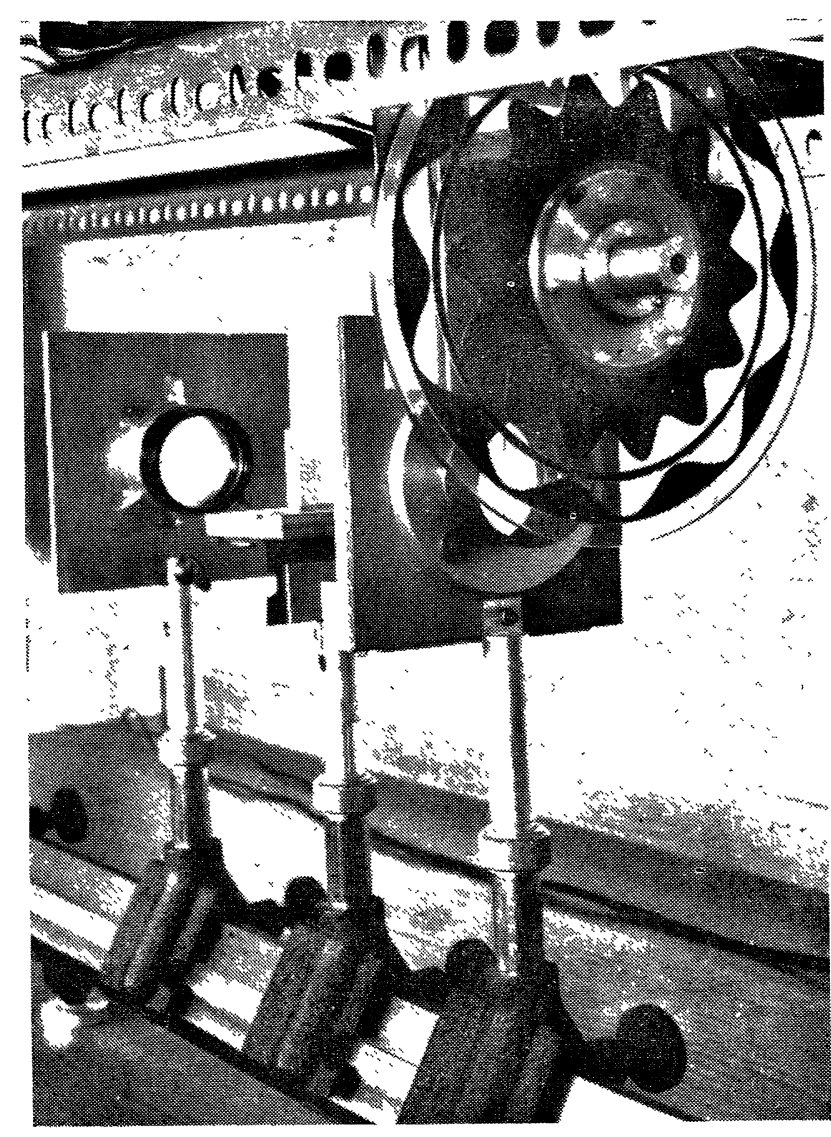

Fig. 3. - Le disque modulateur.

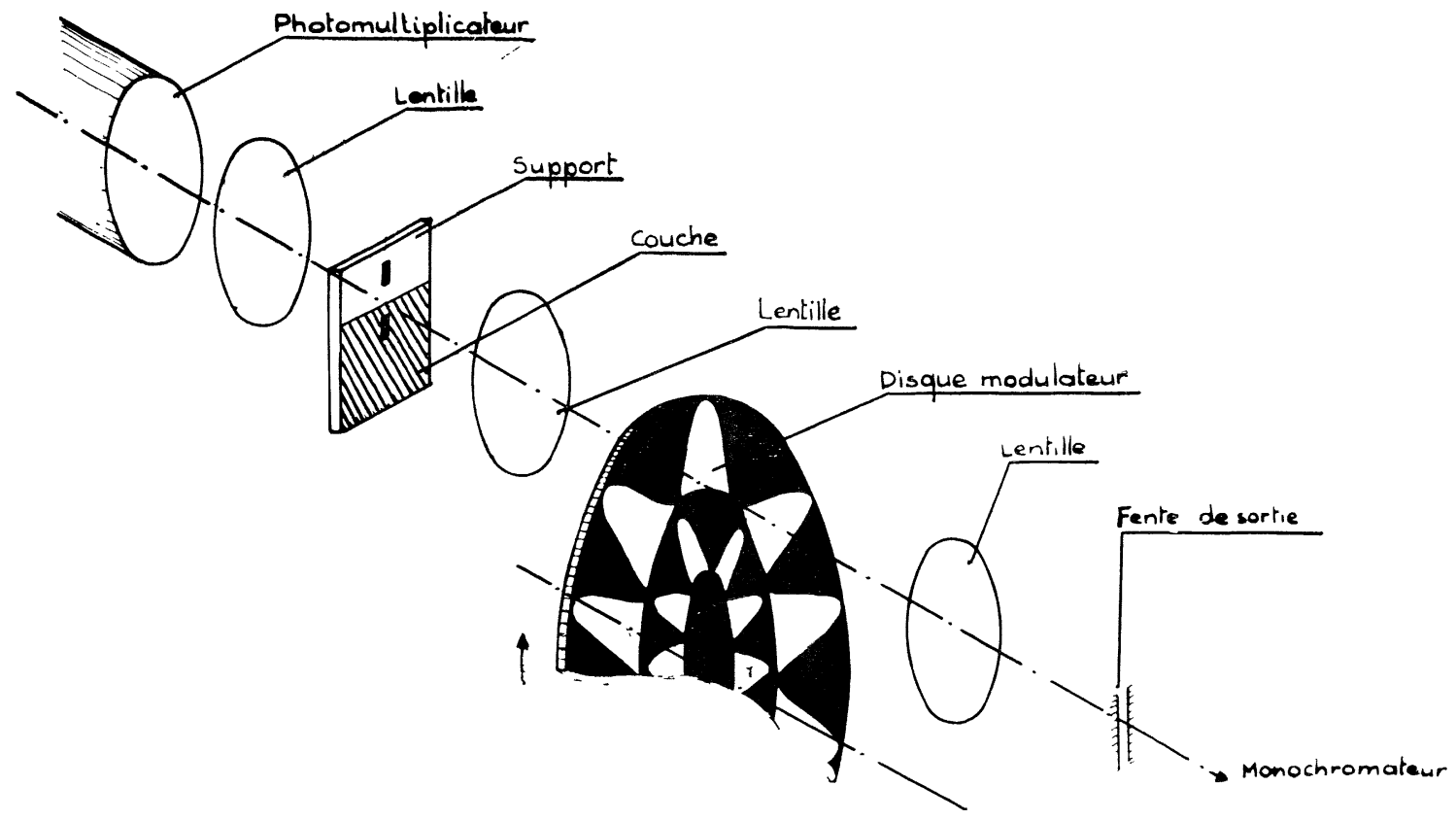

FıG. 4. - Principe de la modulation optique. (Les zones opaques et transparentes sont inversées.) 
de la fente de sortie coïncide avec une portıon d'un rayon du disque ( $f i g .3$ et 4 ). Ce disque, mobile autour de son axe, est entraîné par un moteur asynchrone $e^{-}$syuchronisé alimenté par le secteur $110 \mathrm{~V} 50 \mathrm{~Hz}$. Sur ce disque sont tracées deux courbes concentriques dont les équations sont, en coordonnées polaires centrées sur le centre du disque

$$
\boldsymbol{r}=A+B \cos n \theta
$$

$r$ et $\theta$ désignant respectivement le rayon vecteur et l'angle polaire du point courant. $n$ est un entier dont la valeur est différente pour chacune des deux courbes.

$A$ et $B$ sont des constantes. $B$ a, autant que possible, la même vâleur pour les deux courbes, tandis que $A$ prend deux valeurs différentes telles que la longueur de la portion d'un rayon joignant les deux courbes ne soit en aucun cas inférieure à une longueur donnée (environ $1 \mathrm{~cm}$ ).

Par extension, nous désignerons ces courbes sous le nom de sinusoïdes.

La surface extérieure à la sinusoïde la plus éloignée du centre du disque est rendue opaque à la lumière_ainsi que la surface intérieure à la sinusoïde la plus proche du centre. De plus, une couronne opaque dont la largeur est vorsine de $5 \mathrm{~mm}$ est tracée entre les deux sinusoïdes.

Formons alors l'image de la fente de sortie du monochromateur suivant un rayon du disque et avec un grandissement tel que cette image ait une hauteur suffisante pour couvrir les trois zones opaques quelle que soit la position angulaire du disque.

Supposons que l'image de la fente soit infiniment étroite et qu'elle soit éclairée uniformément sur toute sa longueur par une radiation monochromatique.

Si la vitesse de rotation du disque est de $N$ tours par seconde, nous obtenons ainsi deux faisceaux lumineux dont les flux varient sinusoïdalement (à des constantes additives près qui, après transformation en signaux électrıques, seront facilement éliminables au moyen d'un élément capacitif) aux fréquences $N n_{1}$ et $N n_{2}, n_{1}$ et $n_{2}$ étant les deux nombres entiers correspondant à chacune des sinusoïdes.

L'un de ces faisceaux traverse alors la substance en cours d'étude, l'autre faisceau étant l'étalon. Ensuite, les deux faisceaux sont concentrés, grâce à un système optique, sur la photocathode d'une cellule à multiplicateur d'électrons.

Ainsi, les deux faisceaux de flux $\varphi_{1}$ et $\varphi_{2}$ produisent dans le circuit anodique du photomultiplicateur deux courants de fréquences $N n_{1}$ et $N n_{2}$ et d'amplitudes proportionnelles à $\varphi_{1}$ et à $\varphi_{2}$.

Dans le montage réalisé, nous avons adopté le tracé de deux sinusoïdes comprenant respectivement 10_et 14 périodes complètes. Nous justifierons ce choix au paragraphe $\mathrm{V}$.

Si le moteur était synchrone, sa vitesse de rotation serait de $1500 \mathrm{tr} / \mathrm{mn}$, soit une fréquence de rotation de $25 \mathrm{~Hz}$. On obtiendrait donc des fréquences de modulation de $25 \times 10=250 \mathrm{~Hz}$ et $25 \times 14=350 \mathrm{~Hz}$. En pratique, les fréquences sont légèrement plus basses $(237 \mathrm{~Hz}$ et $334 \mathrm{~Hz}$ pour le matériel dont nous disposions).

Le disque modulateur a été réalisé au moyen d'une graveuse. Lors de la gravure, un moteur électrique imprimait à un disque en plexiglass un mouvement de r'tation uniforme autour de son axe tandis que la fraise était astreinte à suivre un mouvement sinusoïdal dans le temps le long d'un axe fixe situé dans le plan du disque et passant par le centre de ce dernier. Le synchronisme des deux mouvements et le nombre adéquat de périodes complètes étaient obtenus au moyen d'un train d'engrenages de précision.

2. Discrimination et Transformation Des signaux. - Dans la tension que nous obtenons aux bornes de la résistance de charge du photomultiplicateur, il nous faut maintenant prélever les signaux correspondant aux deux faisceaux lumineux, c'est-à-dire les signaux sinusoïdaux de fréquences 237 et $334 \mathrm{~Hz}$. Il nous faut de plus transformer ces signaux afin de les rendre aptes à être enregistrés. Ce résultat est obtenu grâce au montage électronique que nous allons décrire ( $f i g .2$ ).

Ce montage se décompose en deux sous-ensembles désignés par les expressions « Voie 1 » et “ Voie 2 ), ne différant que par les fréquences passantes et rejetées. Nous ne décrirons donc que l'un de ces sous-ensembles.

Si $f_{1}$ et $f_{2}$ sont les fréquences de modulation, la voie électronique mettant en évidence le signal de fréquence $f_{1}$ comporte :

a) Un étage atténuateur et adapteur.

Cet étage a pour but d'affaiblir, dans un rapport variable mais connu avec précision, le signal extrait par la voie considérée et d'adapter l'impédance de sortie à l'impédance relativement faible de l'étage suivant. L'affaiblissement du signal correspondant au flux étalon est en effet nécessaire dans le cas de forte atténuation du faisceau de mesure. Cet étage utilise deux tubes : une double triode ECC83/12AX7 et une triode-pentode ECF82/6U8.

b) Un étage d'amplification sélective sur la fréquence $f_{1}$ et de réjection sur la fréquence $f_{2}$.

L'amplification sélective est obtenue au moyen d'un filtre en double $\mathrm{T}$ monté en contre-réaction entre grille et anode de la partie pentode d'un tube ECF82, la grille écran faisant fonction de grille de commande; la partie triode montée en amplificateur à charge cathodique sert à adapter les impédances.

La réjection se fait au moyen d'un autre filtre en 
double $\mathrm{T}$ attaqué par une triode ECC82/12AU7 montée en charge cathodique.

c) Un étage redresseur.

Afin de pouvoir être mesuré par un enregistreur, le signal fourni par les étages précédents doit être redressé, de façon à fournir un signal continu proportionnel à l'amplitude du signal alternatif.

Une amplification préliminaire (ECC83) amène le signal à un niveau suffisant pour qu'on puisse le redresser linéairement. Le signal attaque alors une triode ECC82 montée en déphaseuse. Une tension redressée en double alternance est enfin obtenue grâce à deux diodes OA85.

d) Un étage d'adaptation en courant continu.

Le signal recueilli sur les cathodes des diodes est envoyé sur un amplificateur à courant continu constitué par une double triode ECC83 montée en charge cathodique symétrique. Cet amplificateur permet d'adapter l'impédance de sortie du montage à l'impédance d'entrée de l'appareil de mesure utilisé (enregistreur potentiométrique ou galvanométrique) et d'effectuer des opérations indispensables de réglage de zéro et de cadrage.

3. Affichage des résultats de mesure. L'enregistrement des résultats de mesure peut s'effectuer de deux manières différentes :

On peut envoyer les deux signaux issus des deux chaînes électroniques sur les deux entrées d'un enregistreur XY. Le balayage en longueur d'onde $\lambda \mathrm{du}$ monochromateur fournit ainsi la courbe d'équations paramétriques

$$
X=k \cdot \varphi_{1}(\lambda) \quad Y=k \cdot \varphi_{2}(\lambda),
$$

$k$ étant une constante.

Mais le résultat généralement utile est le rapport $\varphi_{1} / \varphi_{2}(\lambda)$. Afin d'éviter de fastidieux calculs à l'opérateur, nous avons essayé d'obtenir automatiquement ce quotient. Pour ce faire, nous avons modifié un enregistreur potentiométrique de la manière schématique suivante : La tension de référence appliquée au potentiomètre de l'enregistreur a été remplacée par la tension diviseur, la tension dividende étant envoyée sur l'entrée normale de l'enregistreur. Cette méthode permet théoriquement d'obtenir une lecture proportionnelle au quotient des deux tensions. Les résultats que nous avons obtenus ayant été peu probants, nous pensons nous orienter vers un système utilisant les techniques du calcul analogique.

V. Difficultés de mise au point. - Divers paramètres risquent d'introduire des erreurs dans les résultats que fournit l'appareillage que nous venons de décrire. Nous allons donc analyser les différentes causes d'erreurs possibles.

En premier lieu, la fente de sortie du monochromateur doit fournir un faisceau ayant la même répartition spectrale sur toute sa surface, ce qui peut ne pas être le cas par suite de la présence d'un gradient de température le long de la source. La source doit donc éclairer le monochromateur de façon à ce que cette condition soit réalisée.

D'autre part, les fentes d'entrée et de sortie du monochromateur doivent être parfaitement parallèles, toujours afin d'éviter une différence de répartition spectrale sur les deux faisceaux.

Une répartition non uniforme de l'éclairement de la fente de sortie du monochromateur a pour conséquence la création d'harmoniques dans les signaux recueillis. C'est pour cette raison que nous avons choisi les fréquences de modulations telles que $f_{1} \neq f_{2} \cdot n$ ( $n$ entier ou inverse d'entier).

On peut facilement établir que, dans les conditions où l'on se place, la largeur de la fente n'a qu'une influence négligeable.

Enfin, les étages d'amplification sélective doivent avoir une bande passante dont le sommet soit un méplat afin d'éviter toute variation du gain en cas de fluctuations dans la vitesse de rotation du moteur.

VI. Conclusion. - Si l'on considère le procédé de division des faisceaux, les spectrophotomètres à faisceau double peuvent être répartis en deux classes :

$1^{0}$ Les spectrophotomètres dans lesquels la séparation des faisceaux est obtenue par un diviseur fixe (miroirs, prisme à réflexion totale, ...). De par sa conception, ce type d'appareil nécessite deux détecteurs. Ces détecteurs doivent être placés dans les mêmes conditions ambiantes (température, degré d'humidité, ...) ; ils doivent de plus avoir un comportement identique quant aux effets de fatigue et de vieillissement.

$2^{\circ}$ Les spectrophotomètres utilisant un diviseur rotatif (secteurs tournants, ...) ; un seul détecteur est alors nécessaire. Dans ces appareils, le dispositif d'équilibrage utilise soit la translation d'un coin photométrique, soit le déplacement des lèvres d'une fente collimatrice. Leur construction exige par suite des dispositifs mécaniques usinés avec une grande précision et des asservissements très fidèles.

Nous voyons, grâce à cette brève description des systèmes utilisés en spectrophotométrie automatique, quelles sont les difficultés de réalisation des appareils actuels. La mise en œuvre de la méthode que nous venons de décrire ne présente pas ces difficultés puisque la double modulation des faisceaux permet d'utiliser le même photorécepteur, supprime tout système mécanique, et fournit le découpage des signaux permettant l'emploi d'amplificateurs alternatifs. L'absence de mise au point du dispositif effectuant le quotient ne constitue pas une limitation de la méthode car il existe à l'heure actuelle des quotient-mètres disponibles commercialement.

L’appareillage réalisé ne nous donne cependant 
pas toute satisfaction par suite de fluctuations dues aux nombreuses sources de tension nécessitées par le montage. Il nous est ainsi impossible d'indiquer la précision que l'on peut attendre des mesures effectuées par cette méthode. Nous espérons cependant parvenir à éliminer ces fluctuations et utiliser le spectrophotomètre pour la mesure des paramètres de surfaces ayant subi des traitements multidiélectriques.
VII. Remerciements. - Nous ne saurions terminer sans adresser nos plus vifs remerciements à M. le Professeur P. Bousquet et à M. le Professeur P. Cotton pour les conseils et les encouragements qu'ils nous ont apportés tout au long de ce travail. Toute notre reconnaissance va aussi à M. J. F. Iosca, technicien au laboratoire de l'E. S. P. M., pour son aide amicale lors de la réalisation de cet appareillage.

Manuscrit reçu le 4 août 1965.

\section{BIBLIOGRAPHIE}

[1] GAY (J. Y.), Thèse $3^{e}$ cycle, Marseille, 1964.

[2] Strobex (H. A.), Les méthodes physiques en chimie, Masson et Cie, Paris, 1962.
[3] Terrien 'J.), Baby (P. H.) et Mougeot (P.), Applications industrielles de la spectrophotonétrie. Acta electronica, juillet 1958, vol. $2, \mathrm{n}^{\circ} 3$. 